\title{
DISEÑO Y EVALUACIÓN DE UN PLAN PARA LA ATENCIÓN DEL ALUMNADO INMIGRANTE CON DESCONOCIMIENTO DEL ESPAÑOL
}

\author{
Cecilia Azorín Abellán
}

Universidad de Murcia

\begin{abstract}
RESUMEN: El objetivo general de este artículo es diseñar un plan para la atención del alumnado inmigrante con desconocimiento del español en un Centro de Educación Infantil y Primaria de Murcia. Partiendo de este propósito, los objetivos específicos están asociados a la valoración del proceso de elaboración del plan y a la evaluación del propio plan por parte del personal directivo, docente, orientador e investigador implicado en este proyecto. Para la recogida de información se utilizaron diversas técnicas e instrumentos, entre ellos: un diario de campo y un cuestionario con escala tipo Likert que fue creado ad hoc. En el apartado de resultados, se describen los hallazgos referidos al diseño y a la evaluación del citado plan. Asimismo, se indican los aspectos susceptibles de mejora que fueron recopilados en la memoria de seguimiento, enfocados a: una bienvenida al centro más inclusiva, el uso de la tutoría entre iguales, una mayor inversión en recursos para el apoyo lingüístico y el establecimiento de vínculos con la comunidad. Las conclusiones abordan la necesidad de asumir responsabilidades compartidas para la atención del alumnado inmigrante y desarrollar redes de colaboración entre los distintos agentes que forman parte del contexto educativo y social donde tiene lugar el fenómeno migratorio.
\end{abstract}

PALABRAS CLAVE: Evaluación, educación intercultural inclusiva, estudiantes inmigrantes.

\section{DESIGNING AND EVALUATING A PLAN TO ATTEND TO IMMIGRANT STUDENTS WITH NO KNOWLEDGE OF SPANISH}

\footnotetext{
ABSTRACT: The main aim of this paper is to design a plan of attention for immigrant students with no knowledge of the Spanish language in an Infant and Primary Schoo/ in Murcia, southeast Spain. The specific aims are associated with the evaluation of the preparation of the plan and of the plan itself by the management, teaching, counseling and research persons involved in the project. Information was
} 
collected via various techniques and instruments, including a field diary and an ad hoc Likert type questionnaire. The results section describes the findings regarding the design and evaluation of the plan. Aspects in need of improvement included in the monitoring report are identified and focus on: a more inclusive welcome to the school, the use of peer tutoring, more investment in linguistic support resources and the creation of links with the community. The conclusions address the need to assume shared responsibilities to attend to immigrant students and to develop collaborative networks among the agents that comprise the social and educational context in which the immigration is occurring.

KEYWORDS: Evaluation, inclusive intercultural education, immigrant students.

Recibido: 28/11/2017

Aceptado: 24/01/2018

Correspondencia: Cecilia Azorín Abellán, Universidad de Murcia, Facultad de Educación, Campus Universitario de Espinardo, s/n, 30100 Murcia. Email: cmaria.azorin@um.es.

\section{INTRODUCCIÓN}

La sociedad actual vive inmersa en una crisis humanitaria de refugiados sin precedentes. Según datos de ACNUR (2017), al término de 2016, el número aproximado de personas que se habían desplazado forzosamente de sus lugares de origen por cuestiones relacionadas con persecuciones, conflictos y violaciones de derechos humanos ascendió a 65.6 millones. Esta escandalosa cifra no ha pasado inadvertida en el reciente informe de la OECD (2017) sobre migración internacional, en el que se admite que están Ilegando al continente europeo millares de personas provenientes de países que se encuentran en situación de conflicto. En este sentido, la Comisión Española de Ayuda al Refugiado (2017) destaca la importancia de diseñar procesos de inclusión para personas refugiadas, especialmente en los ámbitos de educación, asistencia social, vivienda, sanidad y empleo. De forma paralela, se ha sumado a esta eventual crisis la tradicional oleada de inmigración en Europa de los últimos años. Desde el punto de vista de los sistemas educativos, este hecho ha incrementado el número de centros escolares que son receptores de estudiantes en distintos momentos del curso académico. Una situación que plantea todo un desafío tanto para el alumnado y sus familias como para las escuelas.

En el contexto español, el intenso flujo migratorio ha propiciado que los centros escolares acojan a estudiantes procedentes de países con diferencias lingüístico-culturales significativas. Evidentemente, esta circunstancia ha generado espacios de multiculturalidad y de reflexión en las aulas (Rosich y López, 2013). Con respecto a la incorporación de estudiantes inmigrantes ${ }^{1}$ a las escuelas, inde-

1 La denominación de "estudiantes inmigrantes" a la que nos referimos se corresponde con la conceptualización propuesta por la Organización para la Cooperación y el Desarrollo Económicos (OECD, 2015b). 
pendiente de que estos sean catalogados como refugiados o no, Garrido (2009, 9) indica lo siguiente:

Cada día son más los alumnos y alumnas inmigrantes que se incorporan a nuestras aulas. Niños y niñas procedentes de distintas latitudes, que están configurando un mapa plural y enriquecedor de los centros educativos, sumamente diferente al de hace tan solo un par de décadas. La escuela se convierte, así, en un escenario excepcional donde recibir, acoger y presentar a estos niños y niñas su nueva realidad; una realidad a la que se acercan con ojos inquietos y curiosos y que, en la mayoría de los casos, no eligieron. El centro escolar y la comunidad educativa que lo integra pueden convertirse en el primer peldaño para que estos niños y niñas asciendan por la escala que supone su integración en nuestra sociedad.

Ineludiblemente, surge en este escenario el interrogante acerca de cómo están respondiendo los diferentes sistemas educativos a los desafíos que plantea la inmigración. Una reflexión que comporta atender a la diversidad del alumnado no solo desde la ética de la eficacia escolar sino también desde el cumplimiento de la educación inclusiva como derecho (Azorín, 2016; Azorín y Ainscow, 2018; Echeita, 2017; Escudero, 2012). Por su parte, Carrasco, Pamies y Narciso (2012) ahondan en la capacidad de los países receptores de inmigración para responder a una composición de alumnado que cada vez es más compleja. En esta dirección, Essomba (2006) alude a la necesidad de liderar escuelas interculturales e inclusivas, poniendo la mirada en los retos que enfrentan los equipos directivos y docentes ante la diversidad cultural y la inmigración. Asimismo, Armstrong y Moore (2004) se refieren a la educación intercultural inclusiva como aquella que exige una visión del hecho educativo centrada en la diversidad y no en la homogeneidad. En última instancia, hay otros referentes teóricos que han abordado las posibilidades y limitaciones de la educación intercultural inclusiva (Moliner, Sales y Escobedo, 2016). Sin embargo, más allá de las bondades que ofrece este modelo, hay que tener en cuenta dos aspectos clave: 1) que la propuesta pedagógica intercultural ha de estar dirigida a toda la comunidad escolar, no únicamente al alumnado de origen inmigrante, y 2) que el proyecto intercultural debe ser concebido como un todo (Leiva, 2015; Walton, Priest y Paradies, 2013).

\section{RetOS QUE AFRONTA LA EDUCACIÓN INTERCULTURAL INCLUSIVA}

En este artículo se describe el proceso seguido durante: 1) la elaboración de un Plan para la Atención del Alumnado Inmigrante con Desconocimiento del Español, que está dirigido a las etapas de Educación Infantil y Educación Primaria (contexto en el que se ha trabajado); y 2) la posterior evaluación que los profesionales implicados en su diseño hacen del mismo. Así pues, en esta sección aparecen algunos de los retos específicos que afronta la educación intercultural inclusiva a la hora de atender a este alumnado, con especial incidencia en las citadas etapas. Para Terrón, Cárdenas y Rodríguez (2017), la educación intercultural inclusiva puede ser considerada como un enfoque de atención y gestión de la diversidad cultural en las instituciones educativas que exige tomar medidas a nivel pedagógico. En esta dirección, algunos de los desafíos que enfrenta este enfoque, en lo que al alumnado inmigrante con desconocimiento del español se refiere, han sido 
expuestos recientemente por Leiva (2017), entre ellos: 1) las dificultades escolares de partida, 2) el desconocimiento del idioma, 3) el aumento excesivo de estudiantes de origen inmigrante en las escuelas públicas, 4) la posible aparición de conflictos religiosos ante determinadas situaciones y 5) la escasa implicación familiar en las tareas escolares.

Por otro lado, aspectos como el nivel socioeconómico, el origen étnico y el estatus migratorio no pueden ni deben determinar la estratificación educativa (Cebolla y Larios, 2009). Considerando en buena medida estos factores, diferentes estudios han concluido que existen desigualdades en los resultados y en las trayectorias educativas del alumnado inmigrante (Calero y Waisgrais, 2009). Incluso se ha llegado a cuestionar si la escuela (concretamente en las etapas de Educación Infantil y Educación Primaria) está preparada para entender y comprender todo aquello que rodea al fenómeno migratorio (García, Granados, Olmos y Martínez, 2014). En España, un dato significativo es que, a pesar de que ha habido un incremento de alumnado inmigrante en la etapa escolar obligatoria de las últimas dos décadas, no se han alcanzado los mismos niveles de incorporación en los estudios postobligatorios. Según expresan Merino y García (2011), los estudiantes inmigrantes luchan más que los estudiantes nativos para completar la educación obligatoria y continuar en la educación postobligatoria. Este hecho sugiere que las medidas que se han tomado para la atención del alumnado inmigrante no están siendo del todo efectivas (Reyes, 2017). Precisamente, el informe que lleva por título Helping immigrant students to succeed at school-and beyond (OECD, 2015a) incide en la necesidad de ayudar a este alumnado para que tenga éxito, no solo en la escuela sino también fuera de ella.

Con relativa frecuencia, algunas de las dificultades que atraviesan los estudiantes cuando asisten a una escuela en un país diferente tienen que ver con el aprendizaje de una lengua, el establecimiento de nuevas amistades y la adaptación a un sistema de educación que no conocen, en un ambiente social que tampoco dominan (Dusi y Steinbach, 2016). Una manera de apoyar a estos estudiantes es a través de la tutoría entre iguales (peer mentoring), una estrategia que está dando muy buenos resultados en los procesos de acogida escolar de países como Inglaterra (Messiou y Azaola, 2017). Asimismo, investigaciones previas en el campo de la acogida educativa han señalado el riesgo de aislamiento al que puede verse expuesto el alumnado inmigrante en aquellas aulas y espacios que han sido diseñados exclusivamente para ellos, con enfoques escolares que en la mayoría de los casos están basados en el modelo del déficit (Etxeberría y Elosegui, 2010; Miravet y García, 2013). En esta línea, existen planteamientos críticos acerca del tipo de respuesta que se facilita al alumnado inmigrante durante su escolarización (Etxeberría y Elosegi, 2009, 21):

La situación de los inmigrantes puede situarse entre la asimilación y la marginación. En otras palabras, estamos atendiendo a un alumnado inmigrante de modo que no le facilitamos la integración escolar y social, empujándolos al fracaso escolar y al desconocimiento de la lengua de acogida, por un lado, y por otra parte a la ruptura con sus señas de identidad, al desprestigio o el abandono respecto a la lengua y cultura familiar. No le enseñamos de modo adecuado lo nuestro, ni les ayudamos a desarrollar lo suyo. No llegamos siquiera a la mera asimilación. La respuesta que les damos se mueve entre la asimilación y la marginación. 
En relación con esto, Messiou (2012) expone en su obra Confronting marginalisation in education. A framework for promoting inclusion un interesante marco de acción para promover la inclusión y reducir la marginalización a la que se ve sometido el alumnado en los contextos educativos. Con respecto a lo anterior, Níkleva (2012) indica que, en la práctica, se están aplicando dos enfoques claramente diferenciados: 1) inclusivo, en el que se trabaja de manera cooperativa con los estudiantes inmigrantes en el aula ordinaria y 2) segregacionista, en el que la atención al alumnado inmigrante se realiza fuera del aula ordinaria. Este dilema ha generado un profundo análisis acerca del tipo de acogida (inclusiva o no) que promueve la normativa vigente. Para Martínez, Fernández y Ayala (2016), dos de las acciones más significativas que propone la actual Ley Orgánica de Mejora de la Calidad Educativa (LOMCE) son la atención a la lengua que porta el alumnado y el cuidado por incluir una educación intercultural en los planes de los centros educativos. Si bien, para que dicha propuesta pueda desarrollarse de manera efectiva, hay que acometer desafíos tales como: 1) transformar las escuelas en instituciones interculturales más justas para todos (Teasley, 2008), y 2) formar en competencias interculturales a los profesionales que se dedican a la acción socioeducativa (Escarbajal y Leiva, 2017).

\section{Método}

\section{Objetivos}

Este artículo recoge las valoraciones que hace el personal de un Centro de Educación Infantil y Primaria (CEIP) que ha participado en la creación y la evaluación de un plan para la atención del alumnado inmigrante con desconocimiento del español.

Así pues, el objetivo general de este trabajo es diseñar un plan para la atención del alumnado inmigrante con desconocimiento del español que inicia su escolaridad en el centro. Partiendo de este propósito principal, los objetivos específicos formulados a este respecto, para la obtención de datos por parte de los profesionales implicados en su elaboración, están asociados a: 1) la valoración del proceso de elaboración del plan y 2) la evaluación del propio plan.

\section{Participantes}

EI CEIP donde se ha desarrollado la investigación está ubicado en la ciudad de Murcia, en un entorno socioeconómico medio-bajo que cuenta con una alta tasa de inmigración en su población circundante. El número de participantes involucrados en el diseño y la evaluación del plan ascendió a 45, considerando los miembros del equipo directivo que colaboraron en este trabajo $\left(n^{2}=3\right)$, el profesorado de la etapa de Educación Infantil $(n=12)$ y Educación Primaria $(n=24)$, la orientadora adscrita al centro $(n=1)$ y el grupo de investigación universitario vinculado a esta propuesta $(n=5)$. Durante la elaboración del plan, se obtuvo asesoramiento por parte del personal que trabaja en el Centro de Animación y Documentación Intercultural dependiente de la CARM (Comunidad Autónoma de la Región de Murcia).

$2 n=$ número de participantes. 


\section{Instrumentos}

En relación con los instrumentos utilizados para la recogida de la información, puede diferenciarse entre: 1) aquellos que sirvieron para valorar el proceso de elaboración del plan (empleados durante la realización y el seguimiento del mismo) y 2) los que permitieron llevar a cabo la evaluación del plan (usados para conocer el grado de satisfacción de los participantes con el producto final creado).

En primer lugar, el equipo investigador hizo uso de un diario de campo, lo que permitió la obtención de anotaciones relevantes durante el proceso de elaboración del plan. Esto facilitó la identificación de aspectos susceptibles de interés sobre las reuniones mantenidas por los participantes y la disposición de otras fuentes de evidencia para la posterior triangulación de la información. También se utilizó un modelo uniforme para la redacción de las actas con la intencionalidad de dejar constancia de los encuentros mantenidos, así como de los temas y acuerdos tratados. Otro instrumento que resultó de utilidad en esta primera fase fue la planilla ideada para el diseño estandarizado del plan.

En segundo lugar, para la evaluación del plan se creó un cuestionario con formato escala tipo Likert (Anexo I) que consta de 40 ítems con 4 opciones de respuesta (en función del grado de acuerdo o desacuerdo) que profundizan en: el proceso de admisión y matrícula (ítems 1 y 2), la información sobre el funcionamiento del centro (ítem 3), la evaluación inicial del alumnado (ítems 4 a 6), el procedimiento de acogida en el grupo-clase de referencia (ítems 7 a 9), la intervención didáctica y los apoyos (ítems 10 a 13), la adaptación del estudiante al aula/centro/comunidad (ítem 14), los recursos humanos y materiales (ítems 15 y 16), la relación con las familias (ítems 17 a 20), la colaboración interinstitucional-local (ítems 21 a 24), la valoración del proceso de acogida (ítem 25) y la propia evaluación del plan (ítems 26 a 40).

\section{Procedimiento y análisis de datos}

El plan de atención del alumnado inmigrante con desconocimiento del español consiste en un documento de referencia para el centro escolar, que está vinculado al proceso de recepción y de atención de dicho alumnado, y parte de una mirada pedagógica intercultural inclusiva. Este plan recoge las actuaciones que se pondrán en marcha desde la escuela para facilitar la adaptación del alumnado de nueva incorporación que presente este perfil.

El plan ha sido diseñado por el equipo directivo y docente del CEIP, junto con la ayuda de la orientadora adscrita al centro y el asesoramiento del personal universitario que ha colaborado en la investigación. Para su correcta coordinación, se programaron una serie de reuniones de trabajo que tuvieron lugar en el primer trimestre del curso escolar 2015/2016. En un primer momento se diseñaron los recursos incardinados al plan de mejora. La siguiente tabla sintetiza los materiales originales que fueron elaborados ad hoc (Tabla 1). 
- Establecer unas orientaciones básicas sobre las acciones a desarrollar para la evaluación inicial del alumnado (idioma español, competencia lingüística y matemática)

$\checkmark$ Concreción del protocolo estándar a seguir durante la evaluación inicial del alumnado por parte del personal implicado.

$\checkmark$ Selección y utilización de pruebas personalizadas para ello.

$\checkmark$ Colaboración con el Centro de Animación y Documentación Intercultural (CADI) y el Servicio de Atención a la Diversidad de la C.A.R.M.

$\checkmark$ Programación de reuniones entre el personal directivo, docente, orientador e investigador para acometer este procedimiento de manera conjunta.

- Recoger por escrito los criterios de admisión y matrícula, así como la información sobre el funcionamiento y organización del centro

$\checkmark$ Revisión de los criterios de admisión.

$\checkmark$ Realización de folletos informativos en distintos idiomas con la organización y funcionamiento del centro.

$\checkmark$ Coordinación con la figura del mediador, si fuera preciso, para ejercer como traductor.

- Definir las funciones del tutor durante el proceso de acogida en el grupo-clase de referencia

$\checkmark$ Redacción de las funciones que debe ejercer el profesor tutor responsable de la acogida en el grupo-clase al que quede adscrito el alumnado.

- Favorecer un proceso de tutoría entre iguales con el alumnado inmigrante

$\checkmark$ Nombramiento de un alumnado tutor que desempeñará el rol de embajador durante el período de adaptación al centro del alumnado inmigrante.

$\checkmark$ Delimitación de las funciones encomendadas al alumnado tutor durante la acogida.

- Consensuar la intervención didáctica y los apoyos que el centro prevé utilizar para responder a la diversidad del alumnado de nueva incorporación con desconocimiento del idioma español

$\checkmark$ Definición de las pautas a tener en cuenta durante la intervención didáctica con este alumnado.

$\checkmark$ Sugerencia y previsión de los apoyos.

$\checkmark$ Elaboración de un programa de inmersión lingüística personalizado para la atención del alumnado con desconocimiento del español.

- Reflexionar sobre el procedimiento de adaptación de este alumnado al aula ordinaria

$\checkmark$ Reunión de seguimiento por parte de los profesionales implicados para concretar cómo se está desarrollando la adaptación del alumnado al aula y al centro.

- Recopilar los recursos humanos y materiales que tiene el centro para atender al alumnado inmigrante

$\checkmark$ Inventario de los recursos materiales del centro para responder a las necesidades del alumnado inmigrante (diccionarios, software, métodos, pruebas de evaluación, y otros).

$\checkmark$ Recopilación de recursos humanos que se pondrán al servicio de este alumnado para su refuerzo y apoyo, dentro y fuera del aula.

$\checkmark$ Utilización de materiales didácticos y creación de recursos propios.

- Colaborar con las familias inmigrantes para su inclusión en la sociedad de acogida, favoreciendo una cercana coordinación con la Asociación de Madres y Padres de Alumnos y Alumnas (AMPA)

$\checkmark$ El Equipo Directivo pondrá en contacto a la familia del nuevo alumnado con la AMPA, favoreciendo el desarrollo de un plan de familias de acogida que colaborarán en la bienvenida y ayudarán en distintas tareas a las familias de nueva incorporación al centro. 
- Delimitar los mecanismos de cooperación que se van a desarrollar dentro del ámbito local con otras instituciones, asociaciones y redes de voluntariado-mediación socioeducativa

$\checkmark$ Apertura del centro hacia la comunidad para sumar apoyos que redunden en una mejora del proceso de acogida del alumnado y de sus familias.

$\checkmark$ Aumento de la interacción con el entorno local (asociaciones, voluntariado).

- Autoevaluar la puesta en marcha del plan, incorporando propuestas de mejora o cambio para el siguiente curso escolar

$\checkmark$ Cumplimentación del cuestionario de evaluación para la valoración del plan.

$\checkmark$ Concreción de propuestas de mejora y renovación/actualización periódica del plan.

RESPONSABLES

- La Comisión de Acogida, formada por el Equipo Directivo y Docente (Equipo de Apoyo a la Diversidad), que contará con la colaboración de la Orientadora del Equipo de Orientación Educativa y Psicopedagógica (EOEP) adscrito, del personal investigador universitario, y de representantes del alumnado, de las familias y de otros agentes de la comunidad.

TEMPORALIZACIÓN

- Tercer trimestre del curso escolar 2015/2016 para la planificación, implementación y evaluación del plan de mejora.

RECURSOS

- Plan de Atención al Alumnado inmigrante.

INSTRUMENTOS:

DE RECOGIDA DE INFORMACIÓN

- Actas de las reuniones acometidas, en cuyo orden del día se indicará cómo está yendo el diseño y desarrollo del plan, los acuerdos tomados y las observaciones que se quiera hacer constar.

- Plan de Trabajo Individualizado adaptado al alumnado de incorporación tardía o compensación educativa, que incluirá información sobre los datos del alumnado (incidiendo en su conocimiento del idioma español-competencia lingüística-, y competencia matemática, así como información relevante acerca de la familia).

- Registro de actuaciones y responsables de la fase informativa, y de acogida.

- Memoria con las propuestas de mejora para el próximo curso escolar.

DE EVALUACIÓN

- Cuestionario de autoevaluación dirigido a los implicados en el plan de mejora realizado al término del tercer trimestre. Este instrumento permitirá evaluar aspectos como el grado de consecución de los objetivos propuestos, la implicación de los responsables, la idoneidad de las actuaciones planteadas, la participación de las familias inmigrantes en el proceso, la coordinación con la administración y el entorno local, y los recursos humanos y materiales con los que se ha contado durante este procedimiento.

Asimismo, se creó un registro de actuaciones y de responsables en el que se explicitó cada una de las actividades de la fase informativa y de la fase de acogida junto con las personas encargadas de realizar las distintas tareas establecidas (Tabla 3). 
Tabla 3. Registro de actuaciones y responsables

\begin{tabular}{|c|c|}
\hline \multicolumn{2}{|c|}{ REGISTRO DE ACTUACIONES Y RESPONSABLES } \\
\hline \multicolumn{2}{|c|}{ FASE INFORMATIVA } \\
\hline Actuaciones & Responsables \\
\hline Admisión y matrícula & Equipo Directivo \\
\hline $\begin{array}{l}\text { Entrevista a la familia para la recogida de da- } \\
\text { tos }\end{array}$ & Equipo Directivo \\
\hline $\begin{array}{l}\text { Información a la familia sobre los aspectos } \\
\text { organizativos del centro }\end{array}$ & Equipo Directivo y tutora \\
\hline \multicolumn{2}{|c|}{ FASE DE ACOGIDA } \\
\hline Actuaciones & Responsables \\
\hline $\begin{array}{l}\text { Preparación de la acogida al aula de referen- } \\
\text { cia }\end{array}$ & Tutora \\
\hline Visita a las dependencias del centro & Tutora y estudiante en el rol de embajador \\
\hline $\begin{array}{l}\text { Presentación de las normas del centro, del } \\
\text { aula y horario }\end{array}$ & Tutora y estudiante en el rol de embajador \\
\hline $\begin{array}{l}\text { Realización de actividades de acogida en el } \\
\text { grupo-clase y de sensibilización con todo el } \\
\text { alumnado (cartel de bienvenida, dinámica de } \\
\text { grupo, murales en dos idiomas) }\end{array}$ & Tutora y grupo-clase de referencia \\
\hline Evaluación inicial & Tutora y orientadora \\
\hline Entrevista a la familia & Tutora \\
\hline $\begin{array}{l}\text { Adecuación de la Programación de Aula e in- } \\
\text { tervención didáctica }\end{array}$ & Tutora \\
\hline Apoyos & Profesorado responsable de ароуо \\
\hline $\begin{array}{l}\text { Previsión de recursos humanos y materiales } \\
\text { necesarios }\end{array}$ & $\begin{array}{l}\text { Equipo Directivo, tutora y orientadora adscri- } \\
\text { ta al centro }\end{array}$ \\
\hline $\begin{array}{l}\text { Actividades interculturales (semana de la di- } \\
\text { versidad, jornadas culturales de puertas abier- } \\
\text { tas, día de las lenguas...) }\end{array}$ & $\begin{array}{l}\text { Comunidad educativa (con la participación } \\
\text { del profesorado, del alumnado y de las fami- } \\
\text { lias) }\end{array}$ \\
\hline $\begin{array}{l}\text { Evaluación del plan de acogida al alumnado } \\
\text { inmigrante }\end{array}$ & $\begin{array}{l}\text { Equipo Directivo y personal investigador uni- } \\
\text { versitario }\end{array}$ \\
\hline
\end{tabular}

Este registro permitió la asignación de tareas a los responsables, lo que favoreció una coordinación efectiva entre los implicados.

El contenido específico del plan (considerando los apartados de los que se compone su índice) quedó configurado de la siguiente manera: 1) introducción al margo legislativo, donde se recoge la legislación autonómica en materia de compensación de desigualdades y atención a la diversidad por la que se rige el plan; 2) información sobre el proceso de admisión y matrícula, en relación con la documentación que debe aportarse para formalizar la matrícula y la recogida de información básica sobre el alumnado y su familia, 3) documentación sobre el funcionamiento y organización del centro, 4) evaluación inicial del alumnado inmigrante con des- 
conocimiento del español, con respecto a la aplicación de pruebas de maduración psicopedagógica, de evaluación de la competencia lingüística y matemática, y de nivel del idioma español; 5) adscripción a un curso, que actuará como grupo-clase de referencia; 6) acogida en el grupo, donde se prevén los preparativos que tendrán lugar para la bienvenida al aula y las funciones que asume el alumnado que ejerce el rol de embajador; 7) intervención didáctica y apoyos, que contempla un programa de inmersión y apoyo lingüístico; 8) adaptación del alumnado inmigrante al aula ordinaria, con respecto al seguimiento de cómo está siendo su proceso de adaptación al grupo-clase; 9) recursos humanos y materiales que se precisan, 10) relación con las familias, apartado en el que se especifican los mecanismos de ayuda y comunicación que el centro pone a su disposición, 11) colaboración con la comunidad local, apartado que tiene como objeto el máximo aprovechamiento de los recursos comunitarios que ofrece el entorno para el alumnado y sus familias, y 12) evaluación del plan, en la que se contempla, entre otros aspectos: el grado de coordinación y participación de los profesionales implicados, la idoneidad de las actividades planteadas, la participación de las familias y la coordinación con los servicios de la comunidad local.

Con respecto al análisis de la información de naturaleza cualitativa, el apartado de resultados contiene una breve síntesis explicativa sobre cómo fue la etapa de planificación. Por último, los datos cuantitativos derivados del cuestionario aplicado fueron volcados en el paquete estadístico SPSS (Statistical Package for the Social Sciences) en su versión 22, lo que permitió la extracción de estadísticos descriptivos básicos (principalmente medias y porcentajes).

\section{Resultados}

Según se ha expuesto anteriormente, durante el diseño del plan se desarrollaron diversas reuniones para revisar la documentación elaborada, así como consensuar las posibles sugerencias de cambio y mejora. En estas sesiones, el equipo investigador recogió información de los asistentes y de los acuerdos tomados. Tal y como se ha detallado, se creó un registro de actuaciones y de responsables en el que se explicitó cada una de las actividades a realizar en las denominadas fase informativa y fase de acogida, junto con las personas encargadas de realizar las distintas tareas establecidas para cada una de ellas. Paralelamente, se adaptó el PTI (Plan de Trabajo Individualizado) propuesto por el Servicio de Atención a la Diversidad de la Consejería de Educación y Universidades de la CARM para su aplicación al alumnado de integración tardía o compensación educativa. A esta plantilla se añadió información relativa a la procedencia familiar, la lengua materna, el conocimiento del español por parte de la familia y el nivel de competencia lingüística y matemática del alumnado. Una vez finalizado el proceso de planificación, se acordó aprobar por unanimidad el plan diseñado en Consejo Escolar para adjuntarlo a la Programación General Anual (PGA). De este modo, el texto elaborado surgió para dar respuesta al alumnado inmigrante con desconocimiento del español escolarizado en el centro y para concretar unas pautas de actuación precisas sobre futuras incorporaciones de otros estudiantes en una situación o perfil similar. 
Posteriormente, tras la planificación del plan y su incorporación a la documentación institucional, se administró un cuestionario al personal directivo, docente, orientador e investigador implicado. El contenido de este cuestionario fue validado mediante un procedimiento interjueces, en el que se contó con la participación de cinco docentes universitarios con experiencia en la construcción de instrumentos y en el ámbito de la interculturalidad. Este cuestionario fue cumplimentado por las 45 personas que tuvieron responsabilidades compartidas en el diseño y el desarrollo del plan. A este respecto, el $100 \%$ de los encuestados manifestó estar de acuerdo y totalmente de acuerdo con los ítems 1 a 25, de lo que se deduce su satisfacción con los puntos abordados en el plan, sobre todo en lo que respecta al proceso de admisión y matrícula; la formación sobre el funcionamiento y organización del centro; la evaluación inicial del alumnado; la acogida del estudiante en el grupo-clase de referencia; la intervención didáctica y los apoyos; la adaptación al aula; los recursos humanos y materiales al alcance; y la colaboración interinstitucional-local. Dentro de los aspectos positivos, un 70\% afirmó que hubo una buena coordinación entre los profesionales y un 65\% indicó que el grado de consecución de los objetivos fue alto. Además, todos los responsables del plan admitieron estar satisfechos con el proceso y resultado del mismo. No obstante, un $80 \%$ reconoció que la participación de las familias en el plan había sido escasa y un $65 \%$ concretó que este tipo de intervenciones tienden a demandar un mayor grado de implicación y de carga temporal.

Por último, se celebró una reunión para la concreción de propuestas de mejora que fueron recogidas en la memoria de seguimiento. Algunas de las propuestas de mejora consensuadas por el CEIP fueron las siguientes:

- Bienvenida inclusiva. De forma previa a la incorporación del alumnado, tanto el centro como el aula de referencia se adornarán con detalles de bienvenida. Con este fin, se introducirán motivos o elementos decorativos acerca del país y la cultura de origen del niño o la niña, favoreciendo así un proceso inicial de acogida más cálido.

- Tutoría entre iguales a partir del rol de embajador. Se determinará al inicio del año académico los alumnos y las alumnas que desempeñarán el rol de embajador o embajadora, así como sus funciones en la acogida del alumnado inmigrante. Para ello, se creará un banco de recursos humanos que estará disponible durante todo el curso escolar.

- Inversión en recursos. Se destinará un presupuesto a la compra de material para la atención del alumnado de compensatoria con y sin desconocimiento del idioma (diccionarios, software, libros, recursos didácticos, pruebas de evaluación de la competencia lingüístico-matemática y enseñanza del español) que quedará a disposición del profesorado en la biblioteca del centro y se incrementarán los medios para desarrollar el programa lingüístico de enseñanza L2.

- Vínculos con la comunidad. Se programarán jornadas de puertas abiertas para el intercambio de experiencias culturales, musicales, lúdicas, gastronómicas o deportivas que permitan estrechar lazos interculturales entre la escuela y la comunidad, las familias, las asociaciones del entorno y otras entidades que colaboran habitualmente con el centro escolar realizando distintas campañas.

Con estas propuestas e iniciativas finales se dio por concluido el proceso. 


\section{Discusión Y CONCLUSIONES}

Retomando el objetivo principal de la investigación, ligado al diseño de un plan para la atención del alumnado inmigrante con desconocimiento del español, puede concluirse que este propósito ha culminado con éxito. No obstante, es importante que el documento creado tenga vida en el centro escolar, que no quede relegado a una estantería y que suponga un verdadero referente sobre el que apoyarse a lo largo del curso académico en aquellos momentos en los que se precise hacer uso de él.

En esta línea, es necesario enfatizar el valor del trabajo realizado para la comunidad educativa más allá del centro en el que se ha desarrollado la presente investigación. Una conclusión que puede ser extensible a diferentes contextos es que la atención del alumnado inmigrante con desconocimiento del español debe asumirse mediante una responsabilidad compartida por parte de la comunidad educativa en su conjunto. Tal y como se ha expuesto en la revisión de la literatura, diversas investigaciones inciden en que la organización de los procesos de acogida en los centros educativos tiene que plantearse de una forma amplia y global, como un reto que incumbe a todo el centro (Garrido, 2009; Leiva, 2015; Simó et al, 2014; Teasley, 2008; Walton, Priest y Paradies, 2013). Sin embargo, la investigación acometida por Etxeberria, Karrera y Murua (2010) evidencia que son muchas las ocasiones en las que el profesorado manifiesta disponer de poco tiempo, recursos, formación y apoyos para enfrentarse a la tarea que requiere la educación con alumnado inmigrante.

A partir del estudio realizado, se ha puesto de manifiesto la importancia que tiene el establecimiento de redes de colaboración formadas por los distintos agentes educativos y sociales de la comunidad. Estas redes han de funcionar de manera efectiva para otorgar, en tiempo y forma, una respuesta ajustada a las necesidades que presenta tanto el alumnado como sus familias. A este respecto, estudios recientes apuestan por el trabajo en red y la construcción de puentes para el apoyo y la colaboración en el ámbito socioeducativo (Azorín, 2017; Azorín y Muijs, 2017). En esta dirección, Simón et al. (2016) concluyen que la posibilidad de tener un espacio para compartir y reflexionar entre centros, intercambiar experiencias y compartir saberes, permite avanzar hacia una educación más inclusiva. En este sentido, la colaboración interinstitucional con otras entidades o agentes, las organizaciones sin ánimo de lucro (no gubernamentales), las asociaciones y las redes de voluntariado constituyen valiosos recursos para la inclusión del alumnado inmigrante en la sociedad de acogida.

A modo de síntesis, otros colegas que han investigado en este campo han formulado propuestas para seguir avanzando hacia un modelo más inclusivo (Simó et al., 2014), entre ellas: 1) las transiciones del aula de acogida al aula ordinaria tienen que ser asumidas de forma coordinada por el equipo docente y no únicamente por el tutor o tutora del aula de acogida, 2) los compañeros y las compañeras del aula ordinaria ejercen un papel fundamental en el seguimiento del aprendizaje de las competencias comunicativas por parte del alumnado inmigrante, 3) la apertura del centro a las familias exige el desarrollo de nuevas formas de relación entre familias heterogéneas para que distintas voces puedan expresar lo que desean y esperan de la escuela, y 4) el profesorado ha de participar en procesos de formación y reflexión sobre otras experiencias similares en contextos educativos diversos. 
Finalmente, somos conscientes de que algunas de las limitaciones que presenta la investigación acometida en este trabajo tienen que ver con su marcado carácter descriptivo, el tamaño de la muestra que ha participado en el diseño y evaluación del plan, y la visión única (posiblemente de carácter parcial y sesgada) que se expone de los profesionales implicados en el proceso. Por consiguiente, siguiendo las directrices de la investigación inclusiva (Nind, 2016), futuros estudios deberán incorporar las distintas voces que constituyen la comunidad, añadiendo a este tipo de investigaciones el testimonio del alumnado, de las familias y de otros agentes que forman parte del contexto educativo y social en el que tiene lugar el fenómeno migratorio.

\section{REFERENCIAS BIBLIOGRÁFICAS}

ACNUR (2017). Tendencias globales. Desplazamiento forzado en 2016. Ginebra: Agencia de la ONU para los Refugiados.

Armstrong, F. y Moore, M. (2004). Action research for inclusive education: Changing places, changing practices, changing minds. London: Routledge Falmer.

Azorín, C. (2016). La respuesta a la diversidad del alumnado en el contexto inglés: un estudio de casos. Enseñanza \& Teaching, 34(2), 77-91. https://doi.org/10.14201/ et20163427791.

Azorín, C. (2017). Redes de colaboración entre escuelas inglesas para la mejora de la inclusión socioeducativa. Profesorado. Revista de Currículum y Formación del Profesorado, Número Extraordinario, 29-48.

Azorín, C. y Ainscow, M. (2018). Guiding Schools on their Journey towards inclusion. International Journal of Inclusive Education, 1-18. https://doi.org/10.1080/13603 116.2018.1450900.

Azorín, C. y Muijs, D. (2017). Networks and collaboration in Spanish education policy. Educational Research, 59(3), 273-296. https://doi.org/10.1080/00131881.20 17.1341817.

Calero, J. y Waisgrais, S. (2009). Factores de desigualdad en la educación española. Una aproximación a través de las evaluaciones de PISA. Papeles de Economía Española, 119, 86-99.

Carrasco, S., Pamies, J. y Narciso, L. (2012). A propósito de la acogida de alumnado extranjero. Paradojas de la educación inclusiva en Cataluña (España). Revista Latinoamericana de Educación Inclusiva, 6(1), 105-122.

Cebolla, H. y Larios, M. J. (2009) Inmigración y educación. Madrid: Centro de Estudios Políticos y Constitucionales.

Comisión Española de Ayuda al Refugiado (2017). Informe 2017: Las personas refugiadas en España y Europa. Madrid: CEAR.

Dusi, P. y Steinbach, M. (2016). Voices of Children and Parents from Elsewhere: A Glance at Integration in Italian Primary Schools. International Journal of Inclusive Education, 20(8), 816-827. https://doi.org/10.1080/13603116.2015.1111948. 
Echeita, G. (2017). Educación inclusiva. Sonrisas y lágrimas. Aula Abierta, 46, 17-24. https://doi.org/10.17811/ri e.46.2017.17-24.

Escarbajal, A. y Leiva, J. J. (2017). La necesidad de formar en competencias interculturales como fundamento pedagógico: Un estudio en la Región de Murcia (España). Profesorado. Revista de currículum y formación del profesorado, 21(1), 281-293.

Escudero, J. M. (2012). La educación inclusiva, una cuestión de derecho. Educatio Siglo XXI, 30(2), 109-128.

Essomba, M. A. (2006). Liderar escuelas interculturales e inclusivas: equipos directivos y profesorado ante la diversidad cultural y la inmigración. Barcelona: Graó.

Etxeberría, F. y Elosegi, K. (2009). Alumnado inmigrante: entre la asimilación y la marginación. Segundas Lenguas e Inmigración en red, 3, 21-41.

Etxeberría, F., Karrera, I. y Murua, H. (2010). Competencias interculturales del profesorado con alumnado inmigrante en el País Vasco. Revista Electrónica Interuniversitaria de Formación del Profesorado, 13(4), 79-94.

García, F. J., Granados, A., Olmos, A. y Martínez, R. (2014). Cuando no hablamos de integración: análisis de la imagen del alumnado "inmigrante" en la prensa en España. Estudios sobre el mensaje periodístico, 20(2), 1081-1101. http://doi. org/10.5209/rev_ESMP.2014.v20.n2.47051.

Garrido, Y. (2009). Puertas abiertas a la igualdad: la acogida del alumnado inmigrante en el centro educativo. Caleidoscopio, revista de contenidos educativos del CEP de Jaén, 2, 9-24.

Leiva, J. J. (2015). Interculturalidad y estilos de aprendizaje: nuevas perspectivas pedagógicas. International Journal of Educational Research and Innovation, 3, 36-51.

Leiva, J. J. (2017). Estilos de aprendizaje y educación intercultural en la escuela. Tendencias pedagógicas, 29, 211-228. http://doi.org/10.15366/tp2017.29.009.

Martínez, M. J., Fernández, C. M. y Ayala, A. (2016). "Yo acojo, tú agrupas, ella compensa": Análisis comparado de la política de integración del alumnado inmigrante en tres Comunidades Autónomas. Revista Complutense de Educación, 27(3), 1103-1118. http://doi.org/10.5209/rev_RCED.2016.v27.n3.47547.

Merino, R. y García, M. (2011). School Dropout and Completion in Spain. En S. Lamb, E. Markussen, R. Teese, N. Sandberg y J. Polesel (Eds.), School Dropout and Completion. International Comparative Studies in Theory and Policy, 99-115. New York: Springer.

Messiou, K. (2012). Confronting marginalisation in education. A framework for promoting inclusion. London: Routledge.

Messiou, K. y Azaola, M.C. (2017). A peer-mentoring scheme for immigrant students in English secondary schools: a support mechanism for promoting inclusion? International Journal of Inclusive Education, 1-16. https://doi.org/10.1080/13603116.2017.1362047.

Miravet, L. M. y García, O. M. (2013). The role of teachers' shared values and objectives in promoting intercultural and inclusive school cultures: a case study. Interna- 
tional Journal of Qualitative Studies in Education, 26(10), 1373-1386. https://doi. org/10.1080/13603116.2017.1362047.

Moliner, O., Sales, A. y Escobedo, P. (2016). Posibilidades y limitaciones de generar cultura de centro desde las prácticas compartidas de aula: el caso de la educación intercultural inclusiva. Estudios Sobre Educación, 30, 51-70. https://doi. org/10.15581/004.30.51-70.

Níkleva, D. (2012). Apoyo Lingüístico para el alumnado extranjero: programas y características. MarcoELE. Revista de Didáctica Español como Lengua Extranjera, 14, 1-7.

Nind, M. (2016). Inclusive research as a site for lifelong learning: Participation in learning communities. Studies in the Education of Adults, 48(1), 23-37. https://doi. org/10.1080/02660830.2016.1155847.

OECD (2015a). Helping immigrant students to succeed at school-and beyond. Paris: OECD Publishing.

OECD (2015b). Immigrant Students at School: Easing the Journey Towards Integration. Paris: OECD.

OECD (2017). International Migration Outlook 2017. Paris: OECD Publishing.

Reyes, C. (2017). Etnografiando formas de aprendizaje, participación y agencia en la escuela y la mezquita. Revista Complutense de Educación, 28(3), 721-735. http:// doi.org/10.5209/rev_RCED.2017.v28.n3.49791.

Rosich, N. y López, P. (2013). La integración del alumnado inmigrante. Estudio comparativo según su procedencia. Números. Revista de Didáctica de las Matemáticas, 84, 47-63.

Simó, N., Pamies, J., Collet, J. y Tort, A. (2014). La acogida educativa en los centros escolares en Cataluña: más allá de los recursos específicos para el alumnado de nueva incorporación. Revista Complutense de Educación, 25(1), 177-194. http:// doi.org/10.5209/rev_RCED.2014.v25.n1.41410.

Simón, C., Sandoval, M., Echeita, G., Calero, C., Nuñez, B., De Sotto, P., Pérez, M. M. y García, A. B. (2016). Transformando la "gramática escolar" para ser más inclusivos: la experiencia de tres centros educativos. Contextos Educativos, 19, 7-24. http://doi.org/10.18172/con.2784.

Teasley, C. (2008). Perspectivas Postcoliniales sobre la Acogida del Alumnado Inmigrante en Galiza, España. Archivos Analíticos de Políticas Educativas, 16(19), 1-36.

Terrón, T., Cárdenas, R. y Rodríguez, R. (2017). Educación intercultural inclusiva. Funciones de los/as educadores/as sociales en instituciones educativas. Pedagogía Social. Revista Interuniversitaria, 29, 25-40. https://doi.org/10.7179/ PSRI_2017.29.02

Walton, J., Priest, N. y Paradies, Y. (2013). Identifying and Developing Effective Approaches to Foster Intercultural Understanding in Schools. Intercultural Education, 24(3), 181-194. https://doi.org/10.1080/14675986.2013.793036. 
Anexo I. Cuestionario para la Evaluación del Plan de Atención al Alumnado Inmigrante (PAAI) con desconocimiento del español

Este cuestionario se ha elaborado con la intencionalidad de evaluar el Plan de Atención al Alumnado Inmigrante con desconocimiento del español. Por favor, valore los aspectos que se enumeran a continuación rodeando con un círculo la respuesta que estime oportuna: (1) totalmente en desacuerdo, (2) en desacuerdo, (3) de acuerdo, y (4) totalmente de acuerdo.

\section{ADMISIÓN Y MATRÍCULA}

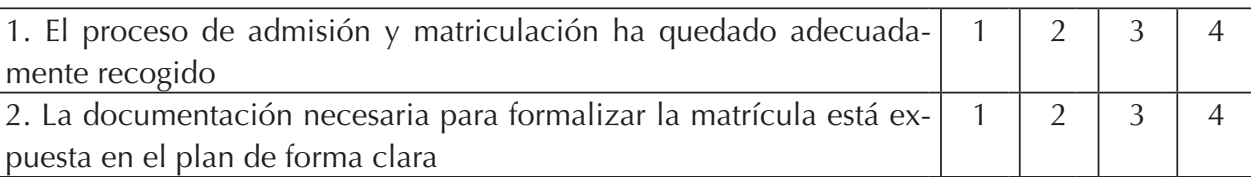

INFORMACIÓN SOBRE EL FUNCIONAMIENTO $\quad \mathrm{Y}$ ORGANIZACIÓN DEL CENTRO

\begin{tabular}{|c|c|c|c|c|}
\hline $\begin{array}{l}\text { 3. La información sobre el funcionamiento y la organización del } \\
\text { centro es accesible para las familias }\end{array}$ & 1 & 2 & 3 & 4 \\
\hline
\end{tabular}

4. El centro dispone de recursos para evaluar el idioma español

\begin{tabular}{|l|l|l|l|l|l}
\hline 5. El profesorado dispone de instrumentos para valorar la competen- & 1 & 2 & 3 & 4
\end{tabular} cia lingüística

\begin{tabular}{|l|l|l|l|l|}
\hline 6. El profesorado dispone de instrumentos para valorar la competen- & 1 & 2 & 3 & 4
\end{tabular}
cia matemática

ACOGIDA DEL ALUMNADO INMIGRANTE EN EL GRUPO-CLASE DE REFERENCIA

\begin{tabular}{|l|l|l|l|l|l}
\hline 7. El plan diseñado prevé cómo será la bienvenida del alumnado & 1 & 2 & 3 & 4
\end{tabular} inmigrante

\begin{tabular}{|l|l|l|l|l|}
\hline 8. Se han delimitado las funciones del alumnado-tutor que ejercerá & 1 & 2 & 3 & 4
\end{tabular} el rol de embajador

\begin{tabular}{|l|l|l|l|l|}
\hline 9. Se ha concretado el papel que desempeñará el maestro-tutor du- & 1 & 2 & 3 & 4
\end{tabular} rante el proceso de acogida

INTERVENCIÓN DIDÁCTICA Y APOYOS

\begin{tabular}{|l|l|l|l|l|}
\hline 10. Se ha establecido un plan de intervención didáctica (programa & 1 & 2 & 3 & 4
\end{tabular} de inmersión lingüística) para el aprendizaje del español en el alumnado con desconocimiento del idioma

\begin{tabular}{|l|l|l|l|l|}
\hline 11. Se ha consensuado la participación del alumnado inmigrante en & 1 & 2 & 3 & 4
\end{tabular} áreas que favorezcan su integración y socialización inicial (Educación Física, Educación Artística, Inglés)

\begin{tabular}{|l|l|}
\hline 12. Se ha estimado oportuno que el alumnado que lo requiera asista & 1
\end{tabular} a clases de refuerzo de lengua y matemáticas en niveles inferiores 13. Se han organizado las bases que regirán el proceso de apoyo al alumnado inmigrante con desconocimiento del idioma que se incorpora al centro

ADAPTACIÓN DEL ALUMNADO AL AULA-CENTRO-COMUNIDAD

14. Se han consensuado unas pautas de adaptación para la inclusión del alumnado inmigrante en el aula, en el centro, y en la comunidad 


\begin{tabular}{|c|c|c|c|c|}
\hline \multicolumn{5}{|l|}{ RECURSOS HUMANOS Y MATERIALES } \\
\hline $\begin{array}{l}\text { 15. El plan contempla los recursos humanos que se precisan para la } \\
\text { atención del alumnado inmigrante y de sus familias }\end{array}$ & 1 & 2 & 3 & 4 \\
\hline $\begin{array}{l}\text { 16. El plan recoge los recursos materiales con los que cuenta el cen- } \\
\text { tro para responder a la diversidad del alumnado inmigrante }\end{array}$ & 1 & 2 & 3 & 4 \\
\hline \multicolumn{5}{|l|}{ RELACIÓN CON LAS FAMILIAS } \\
\hline $\begin{array}{l}\text { 17. El plan establece cómo será la relación del centro con las fami- } \\
\text { lias }\end{array}$ & 1 & 2 & 3 & 4 \\
\hline $\begin{array}{l}\text { 18. El plan introduce la participación activa de la Asociación } \\
\text { de Madres y Padres de Alumnos y Alumnas en el proceso de } \\
\text { acogida }\end{array}$ & 1 & 2 & 3 & 4 \\
\hline $\begin{array}{l}\text { 19. Se ha considerado el nombramiento de familias tutoras colabo- } \\
\text { radoras para el desempeño de una labor de tutoría voluntaria con las } \\
\text { familias inmigrantes de nueva incorporación al centro }\end{array}$ & 1 & 2 & 3 & 4 \\
\hline 20. El plan recoge cómo será la relación del tutor y la familia & 1 & 2 & 3 & 4 \\
\hline \multicolumn{5}{|l|}{ COLABORACIÓN INTERINSTITUCIONAL-LOCAL } \\
\hline $\begin{array}{l}\text { 21. El plan alude a la coordinación del centro con la comunidad } \\
\text { local }\end{array}$ & 1 & 2 & 3 & 4 \\
\hline $\begin{array}{l}\text { 22. El plan promueve la colaboración del centro con asociaciones y } \\
\text { otras instituciones socioeducativas del entorno }\end{array}$ & 1 & 2 & 3 & 4 \\
\hline $\begin{array}{l}\text { 23. El plan describe la importancia del voluntariado en el proceso } \\
\text { de acogida }\end{array}$ & 1 & 2 & 3 & 4 \\
\hline $\begin{array}{l}\text { 24. El plan incorpora la figura del mediador intercultural y su cone- } \\
\text { xión con el centro escolar }\end{array}$ & 1 & 2 & 3 & 4 \\
\hline \multicolumn{5}{|l|}{ VALORACIÓN DEL PROCESO DE ACOGIDA } \\
\hline $\begin{array}{l}\text { 25. El plan aborda mecanismos para la evaluación trimestral del } \\
\text { alumnado de acogida por parte del equipo directivo y docente }\end{array}$ & 1 & 2 & 3 & 4 \\
\hline \multicolumn{5}{|l|}{ EVALUACIÓN GLOBAL DEL PLAN ACOMETIDO } \\
\hline 26. El plan ha sido fruto de la labor de todo el profesorado del centro & 1 & 2 & 3 & 4 \\
\hline $\begin{array}{l}\text { 27. Los profesionales implicados en el plan se han mostrado com- } \\
\text { prometidos }\end{array}$ & 1 & 2 & 3 & 4 \\
\hline 28. El material utilizado para elaborar el plan ha sido adecuado & 1 & 2 & 3 & 4 \\
\hline $\begin{array}{l}\text { 29. Durante la realización del plan ha habido intercambio de ideas, } \\
\text { información y materiales }\end{array}$ & 1 & 2 & 3 & 4 \\
\hline $\begin{array}{l}\text { 30. La coordinación entre los profesionales que se han encargado de } \\
\text { Ilevar a cabo el plan ha sido buena }\end{array}$ & 1 & 2 & 3 & 4 \\
\hline 31. Se ha escuchado la opinión de todos los implicados en el plan & 1 & 2 & 3 & 4 \\
\hline 32. El plan ha partido del diálogo, la negociación y el consenso & 1 & 2 & 3 & 4 \\
\hline $\begin{array}{l}\text { 33. Se ha dispuesto de tiempo suficiente para elaborar y revisar el } \\
\text { plan }\end{array}$ & 1 & 2 & 3 & 4 \\
\hline $\begin{array}{l}\text { 34. Se ha trabajado de manera efectiva para la inclusión del alumna- } \\
\text { do de acogida en la dinámica del centro }\end{array}$ & 1 & 2 & 3 & 4 \\
\hline $\begin{array}{l}\text { 35. El grado de consecución de los objetivos del plan de acogida ha } \\
\text { sido alto }\end{array}$ & 1 & 2 & 3 & 4 \\
\hline
\end{tabular}




\begin{tabular}{|l|c|c|c|c|}
\hline $\begin{array}{l}\text { 36. Las actuaciones previstas dentro del plan han resultado útiles } \\
\text { para su ejecución }\end{array}$ & 1 & 3 & 4 \\
\hline $\begin{array}{l}\text { 37. Las familias inmigrantes han participado activamente en el plan } \\
\text { de acogida }\end{array}$ & 1 & 2 & 3 & 4 \\
\hline $\begin{array}{l}\text { 38. Los recursos humanos previstos en el plan han sido suficientes } \\
\text { para su adecuada puesta en marcha }\end{array}$ & 1 & 2 & 3 & 4 \\
\hline $\begin{array}{l}\text { 39. Los recursos materiales recopilados en el plan han resultado su- } \\
\text { ficientes }\end{array}$ & 1 & 2 & 3 & 4 \\
\hline $\begin{array}{l}\text { 40. En líneas generales, estoy satisfecho/a con el proceso y resultado } \\
\text { del plan }\end{array}$ & 1 & 2 & 3 & 4 \\
\hline
\end{tabular}

